Musées, Patrimoine et Culture scientifiques et techniques

\title{
Le Jardin botanique de Lyon : d'un service technique à un musée vivant
}

\author{
entretien avec Frédéric Pautz
}

\section{OpenEdition \\ Journals}

Édition électronique

URL : http://journals.openedition.org/ocim/1516

DOI : $10.4000 /$ ocim. 1516

ISSN : 2108-646X

\section{Éditeur}

OCIM

\section{Édition imprimée}

Date de publication : 1 mai 2015

Pagination : 20-25

ISSN : 0994-1908

\section{Référence électronique}

entretien avec Frédéric Pautz, « Le Jardin botanique de Lyon : d'un service technique à un musée vivant », La Lettre de I'OCIM [En ligne], 159 | 2015, mis en ligne le 01 mai 2016, consulté le 20 avril 2019. URL : http://journals.openedition.org/ocim/1516 ; DOI : 10.4000/ocim.1516

Ce document a été généré automatiquement le 20 avril 2019.

Tous droits réservés 


\section{Le Jardin botanique de Lyon : d'un service technique à un musée vivant}

entretien avec Frédéric Pautz

NOTE DE L'ÉDITEUR

Entretien réalisé par Olivier Soichot

La grande serre du Jardin botanique de Lyon

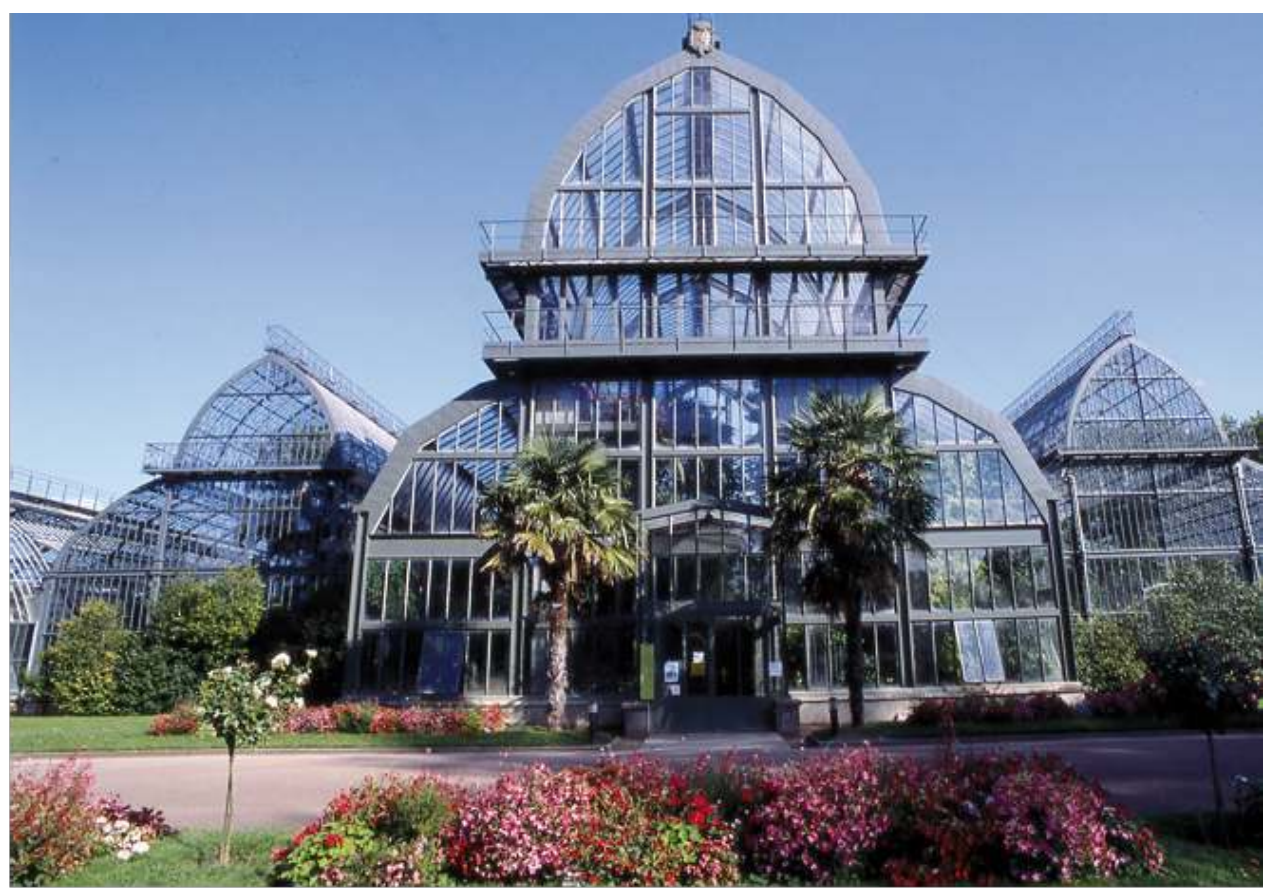

(C) Jardin botanique de Lyon 


\section{Pouvez-vous nous présenter le Jardin botanique de Lyon, son histoire et ses missions institutionnelles ?}

1 Le Jardin botanique de Lyon est un jardin créé pendant la Révolution Française, en 1796 précisément. Au départ situé dans le centre historique de Lyon, sur les Pentes de la Croix Rousse, il a ensuite été transféré au Parc de la Tête d'Or en 1857, site qu'il occupe maintenant depuis près de 160 ans. À l'époque de ce déménagement, le jardin porte le nom de Jardin des Plantes. C'est en 1859 qu'il devient le Jardin botanique.

2 Le Jardin botanique de Lyon a grandi au cours du temps. Il dispose aujourd'hui de collections patrimoniales très importantes réparties sur 8 hectares dont 33 serres recouvrant $7200 \mathrm{~m}^{2}$. Le Botanic Gardens Conservation International (BGCI), réseau mondial des jardins botaniques, le classe d'ailleurs à l'échelle mondiale parmi les dix plus grands jardins botaniques en termes de nombre de taxons. Cela représente à peu près 15000 plantes différentes, botaniques et horticoles, représentant 22000 accessions (numéros d'inventaire).

3 C'est un jardin extrêmement varié dont les collections vont des plantes de montagne aux roses en passant par les aracées tropicales, les orchidées, les palmiers, la flore locale française, les arbustes...

L'intérieur de la serre de Madagascar.

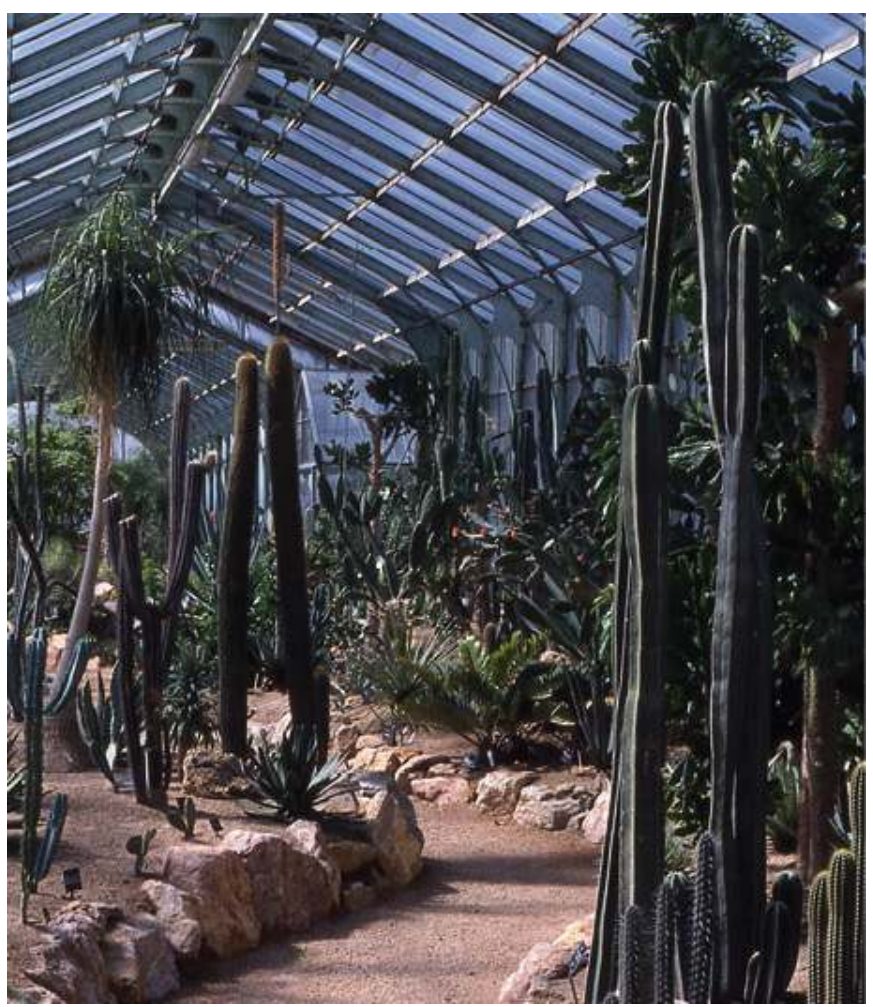

(c) Jardin botanique de Lyon/L. Carré

Parmi ces collections très éclectiques, le Jardin botanique de Lyon est également considéré comme référent à l'échelle nationale pour une dizaine d'entre elles (bégonias botaniques, plantes carnivores, aracées, clématites, pivoines, dahlias) par le 
Conservatoire des collections végétales spécialisées (CCVS), une association dont l'objectif est de fédérer toutes les initiatives privées ou publiques visant à préserver le patrimoine horticole et botanique vivant. Dans la famille des aracées, David Scherberich, notre agent responsable de la collection, participe activement à des missions internationales pour collecter et découvrir de nouvelles espèces. Il en est de même pour plusieurs de ses collègues.

En dehors du patrimoine botanique vivant, le Jardin botanique de Lyon dispose aussi d'un fonds d'herbiers comprenant 270000 parts, dont 530 types nomenclaturaux déjà identifiés à ce jour. On continue d'en découvrir de nouveaux chaque jour. Les plus vieux datent de 1635. Nous disposons aussi d'un fonds documentaire d'environ 8500 ouvrages dont 400 antérieurs au XVIII ${ }^{e}$ siècle. Le jardin est abonné à 70 périodiques et participe à des réseaux nationaux d'échanges de documentation.

6 En matière d'infrastructures, le Jardin botanique de Lyon présente des serres différentes qui permettent de recréer de nombreux climats du monde. Il existe cinq groupes de serres : la serre hollandaise, les grandes serres, les petites serres chaudes et froides, la serre Victoria et la serre de Madagascar. L'une de nos structures les plus remarquables est la grande serre historique, inscrite à l'inventaire des Monuments historiques. Avec son dôme en ogive culminant à 21 mètres, c'est la plus haute de France et l'une des plus hautes d'Europe. Construite entre 1877 et 1882, cette grande structure de verre et d'acier est l'une des rares en France de cette taille à avoir perduré grâce notamment à plusieurs actions de rénovation et d'entretien au cours de son histoire. Ces interventions ont lieu tous les 30 ans et nécessitent de gros travaux.

7 Pour assurer son fonctionnement, le Jardin botanique de Lyon comprend une équipe de 36 personnes dont 19 «jardiniers botanistes » qui font partie de ces nouveaux métiers émergents avec des missions bien spécifiques dans le domaine de la préservation et de la conservation du patrimoine botanique. Le jardin dispose aussi d'un pôle médiation et communication ( 7 personnes dont 6 temps plein), un pôle recherche et scientifique (5 personnes), un pôle ressources chargé du fonds documentaire (3 personnes), et enfin une direction (2 personnes).

Sur le plan statutaire, le Jardin botanique de Lyon est rattaché à la direction des espaces verts de la ville et non pas aux affaires culturelles comme cela est le cas dans plusieurs autres villes de France. Ce rattachement à l'aménagement urbain est un aspect important puisqu'il permet au jardin de profiter du savoir-faire d'un service technique (matériel à moteur et outils, logistique, centre de production de plantes, techniques horticoles). C'est l'un des sites de la direction espaces verts qui gère des collections végétales vivantes. Cependant, à travers ses missions fondamentales, édicté par convention avec le réseau mondial des jardins botaniques (BGCI), c'est aussi un musée vivant, qui à pour vocation la recherche, l'éducation et la sauvegarde du patrimoine végétal. Dans ce sens, le jardin est vraiment à l'interface entre ces deux sensibilités et organisations.

Concrètement, cela implique que nous travaillons en synergie avec d'autres musées de la ville dans le cadre de manifestations. C'est le cas pour le prochain Congrès mondial des Sociétés de Roses prévu en juin 2015 (lien avec le musée Gadagne, le musée de l'Imprimerie, les archives municipales et le musée des Beaux-Arts). À titre d'exemple, en 2003, nous avions aussi coordonné avec 45 partenaires la conception de l'exposition Hommes, plantes, insectes, qui a rassemblé en 3 mois plus de 160 événements dans l'agglomération lyonnaise. 
Une collection importante de plantes : ici le Hoya imperialis.

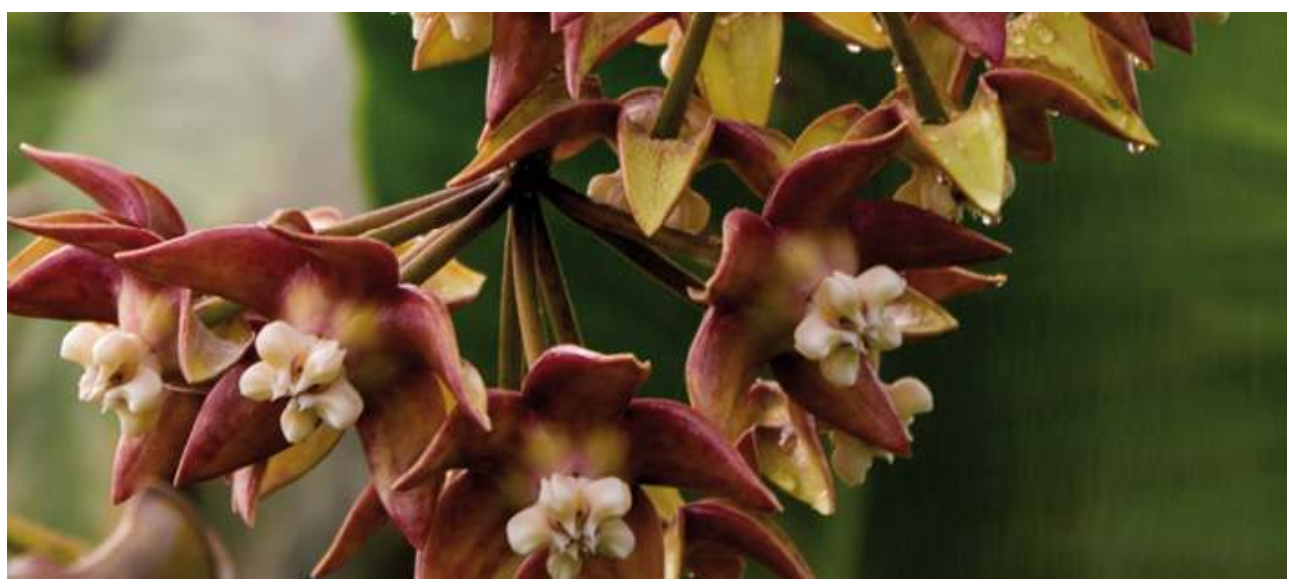

(c) Jardin botanique de Lyon/L. Carré

Sur le plan de la conservation et de la valorisation, le Jardin botanique de Lyon entretient aussi des liens avec l'université de Lyon (le PRES de Lyon) ou d'autres instances comme l'Union internationale pour la conservation de la nature (UICN), le CCVS ou le réseau national des jardins botaniques (JBF).

Une serre de culture et de multiplication.

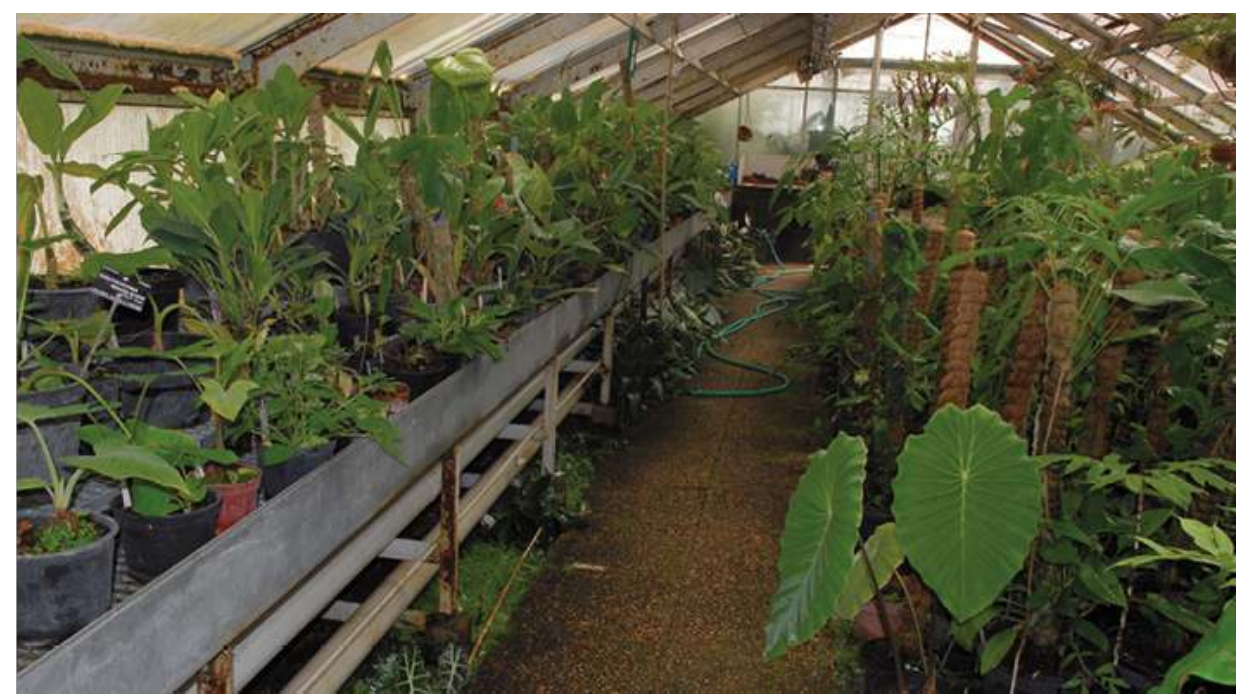

(c) Jardin botanique de Lyon

\section{Concernant la mission de conservation du jardin, quel est votre périmètre d'intervention au niveau des collections?}

11 S'agissant des types de plantes que l'on protège, celles-ci se découpent en trois grandes catégories. La première concerne les espèces liées à l'UICN, c'est-à-dire la flore tropicale et tempérée à l'échelle mondiale. Cela représente 1200 taxons de plantes menacées de disparation (dont 30 considérées comme disparues dans la nature). À travers le réseau 
international du BGCI, nous participons à la mise en œuvre de la Convention sur la diversité biologique et fournissons du matériel vivant pour de nombreux chercheurs. Nous recevons plusieurs sollicitations par semaine dans ce sens.

Notre second niveau d'implication porte sur la flore locale, en lien avec les conservatoires botaniques nationaux (les CBN) et les gestionnaires d'espaces naturels pour la sauvegarde de plantes de la région Rhône-Alpes, au niveau de l'agglomération lyonnaise tout du moins. Ainsi, entre 2007 et 2011, les équipes du jardin ont participé activement à la cartographie de la flore de l'ensemble du territoire de la métropole, sur des mailles de $1 \mathrm{~km}$ sur $1 \mathrm{~km}$.

13 Enfin, le dernier niveau a trait à la flore horticole. Au XIX ${ }^{\mathrm{e}}$ siècle, Lyon était une grande capitale de l'horticulture comme l'attestent beaucoup d'ouvrages publiés sur le sujet. Sur ce plan, notre mission porte sur la sauvegarde des fruits, des fleurs et des légumes qui ont été créés dans l'agglomération lyonnaise. Ces variétés locales constituent aujourd'hui un élément patrimonial de première importance.

\section{Le Jardin botanique de Lyon est aussi au cœur d'un grand chantier de rénovation. Quand a-t-il commencé ?}

Cette rénovation s'inscrit dans une démarche qui date de plusieurs années et qui répond à des considérations d'ordre interne, mais aussi à toute une série de textes nationaux et internationaux. À l'image de ce qui s'est passé dans les muséums d'Histoire naturelle pour organiser et valoriser leurs collections, nous avons donc entamé une mutation pour passer d'un endroit qui « amasse » des collections et propose du service technique à un véritable musée vivant. Clarifier nos missions, définir nos stratégies, mettre en adéquation les missions et les collections vivantes fait partie des axes de notre réflexion et de nos actions. Il faut donner du sens à nos collections et en particulier donner plus de lisibilité à nos actions auprès des 450000 personnes qui nous rendent visite chaque année.

Depuis 15 ans, l'organigramme a beaucoup évolué et de nombreux nouveaux métiers ont émergé : la création du pôle médiation et tout ce qu'une politique en faveur des publics implique, la valorisation scientifique des herbiers et des collections vivantes, l'ouverture du fonds documentaire, la création de deux grandes expositions annuelles, l'implication et la visibilité sur Internet (www.jardin-botanique-lyon.com).

Au niveau de la direction du jardin, nos efforts visent à faire perdurer cette dynamique du passage d'espace technique à musée vivant tant au niveau des infrastructures que de nos missions. Ainsi, nous espérons poursuivre ce programme avec la rénovation des petites serres horticoles. L'idée serait de transformer ces $2000 \mathrm{~m}^{2}$ de collections en espaces scénographiés avec des thématiques répondant à des préoccupations environnementales modernes, un message clair, plus d'interactivité pour les publics (adultes, enfants, familles, spécialistes, publics spécifiques)...

17 Au final, à travers ces questionnements, nous construisons un plan d'action pluriannuel au service de nos objectifs. 


\section{Comment cette rénovation réoriente-t-elle l'organisation de l'équipe et ses compétences ?}

Cette transformation induit plusieurs étapes au niveau du personnel. Nos jardiniers constituent un personnel très spécialisé et hautement qualifié. Pour la plupart, il s'agit de personnes passionnées, qui voyagent à travers le monde pour chercher des plantes et parfaire leurs connaissances. Le Jardin botanique de Lyon a donc cette chance de disposer d'agents ayant une grande plus-value au niveau des compétences et de l'investissement qu'ils affichent dans l'accomplissement de leurs missions.

À partir de là, il était pertinent de lancer un audit interne afin de recueillir les idées de l'ensemble du personnel par rapport à cette démarche de rénovation. Parallèlement, une étude a été lancée en 2014 pour nous aider à formaliser nos idées et nous enrichir d'un regard extérieur. Enfin, pour ma part, j'ai également eu la chance de voyager dans de très nombreux pays et de me nourrir, à travers ces rencontres, des expériences et de multiples points de vue alimentant notre réflexion. Actuellement, nous sommes dans une phase de synthèse entre la vision des agents, la mienne et celle apportée par l'étude.

Pour illustrer l'impact qu'a eu cette rénovation au niveau des équipes, il suffit de regarder les changements opérés au niveau du temps de travail des agents. Lorsque nous étions un service purement technique, les agents travaillaient du lundi au vendredi. Or, la fréquentation du jardin (450 000 visiteurs/an pour les serres) se fait essentiellement le week-end. Nous avons donc dû repenser toute notre organisation pour adapter les temps de travail de notre personnel aux besoins d'un musée et donc de la population. Ces mutations sont en cours. Nous sommes un service public et nous devons repenser notre travail afin de répondre aux attentes du public.

21 De la même façon, le travail de nos jardiniers a évolué au cours de ces quinze dernières années. Aujourd'hui, nos agents ont complètement intégré le travail de valorisation de ce patrimoine dans leur cœur de métier. Le travail ne se limite pas à maintenir en vie les collections vivantes. À travers des visites guidées, des animations, l'écriture de textes destinés aux expositions, la réalisation de photographies, ou encore la rédaction d'articles dans notre revue Sauvages et Cultivées, tous sont des jardiniers, mais aussi par moment des botanistes ou des médiateurs. Régulièrement, ils sont aussi amenés à établir des partenariats avec d'autres jardins ou rencontrer d'autres collègues au niveau national pour diversifier et enrichir leurs connaissances. Tout cela demande des agents d'une extrême compétence. Ils sont fortement impliqués dans la gestion de leurs collections et ces responsabilités sont pour eux très stimulantes.

$\mathrm{Au}$ niveau de la direction, tout le travail de recrutement et de management que nous menons depuis une quinzaine d'années va dans ce sens. La fiche de poste de jardinier botaniste mentionne aussi clairement toutes ces compétences.

\section{Cette évolution des métiers est-elle aussi perçue au niveau du public du jardin?}

23 Je pense que les choses avancent mais c'est un travail de longue haleine. Pour faire évoluer les mentalités, nous misons beaucoup sur la poursuite de la rénovation du jardin. De la même façon, nous essayons de faire passer ce message dans notre communication : à 
travers notre site Internet et les réseaux sociaux ou encore sur des actions in situ comme nos visites guidées par exemple. Grâce à tout cela, nous avançons progressivement vers une meilleure lisibilité du jardin par la population.

L'animation scolaire : « Sur la trace des arbres ».

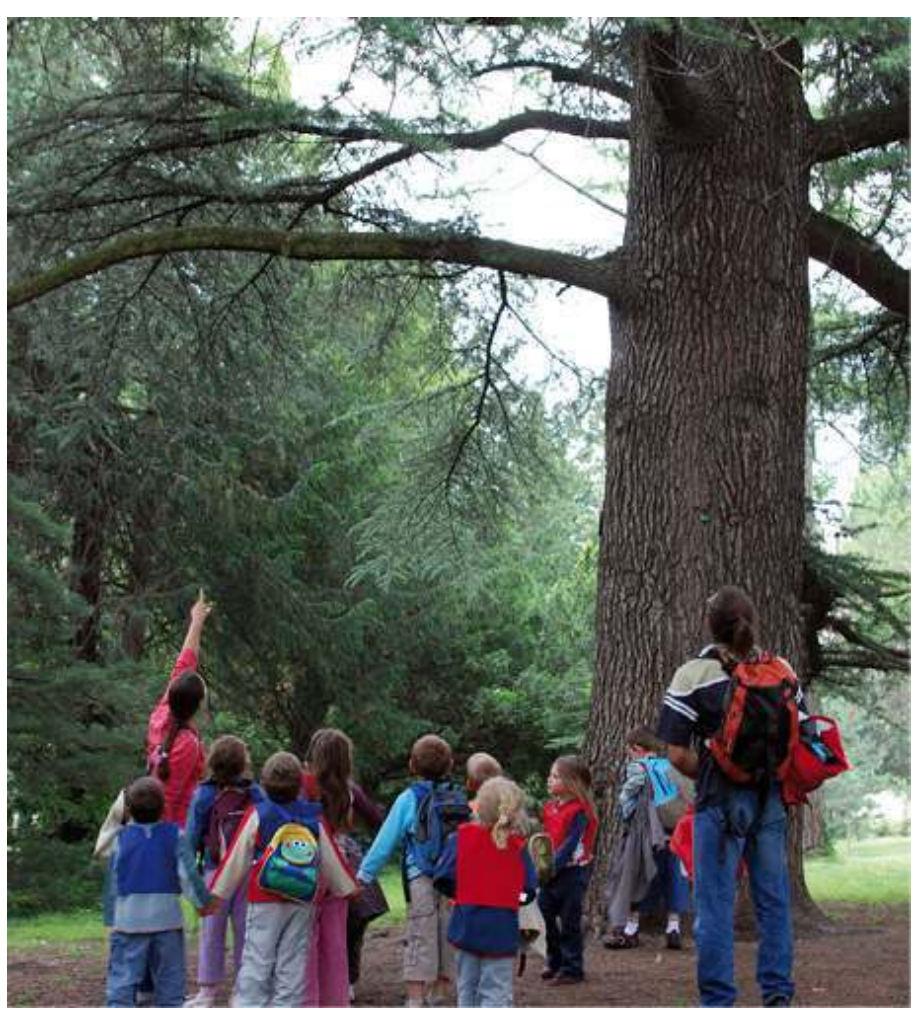

(c) Jardin botanique de Lyon 
L'atelier pour adultes : « La culture des plantes succulentes ».

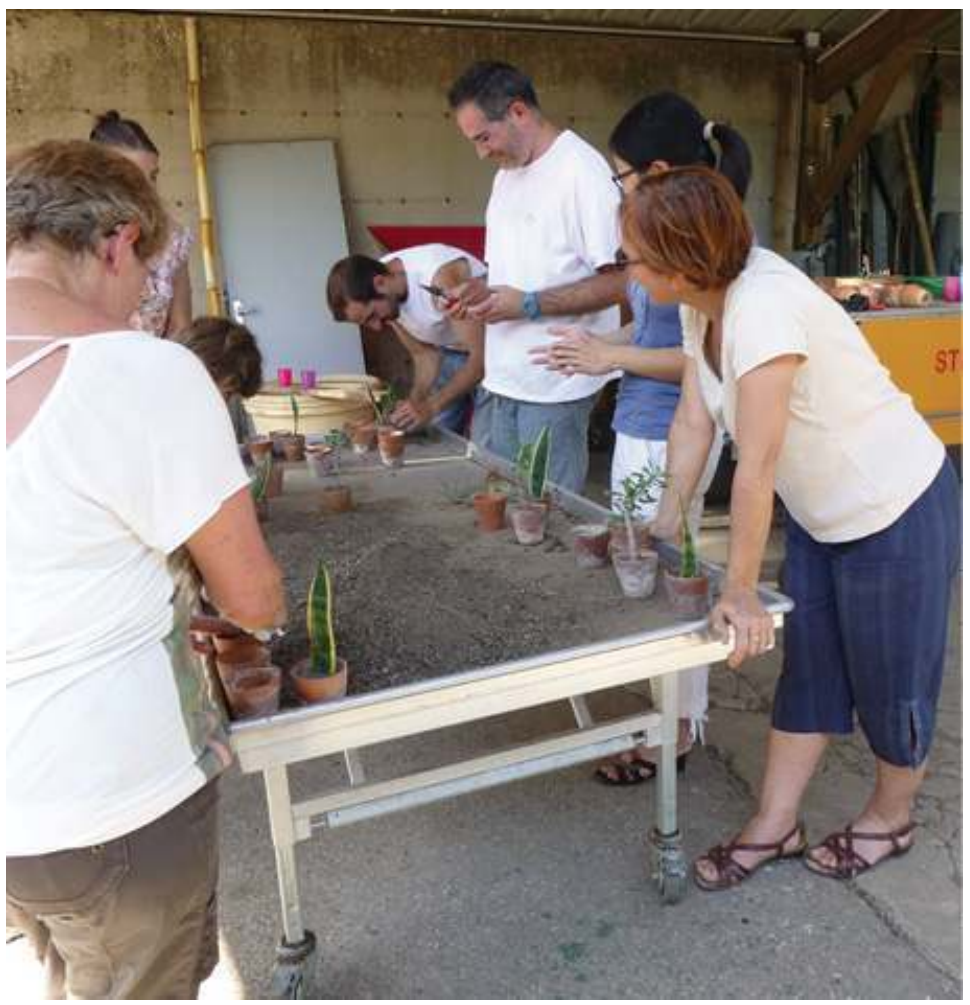

(c) Jardin botanique de Lyon/F. Eyzat

\section{Pour mieux cerner les besoins de cette population, avez-vous réalisé des enquêtes de publics?}

Oui, en 2005, nous avons lancé une enquête auprès de 1000 personnes, dont un tiers a été interrogé au sein du Jardin botanique et du Parc de le Tête d'Or, un tiers place Bellecour, et un tiers autour de la gare de la Part Dieu. Nous avons collecté des résultats intéressants mais pas forcément sur les services attendus. 
Le pavillon des expositions.

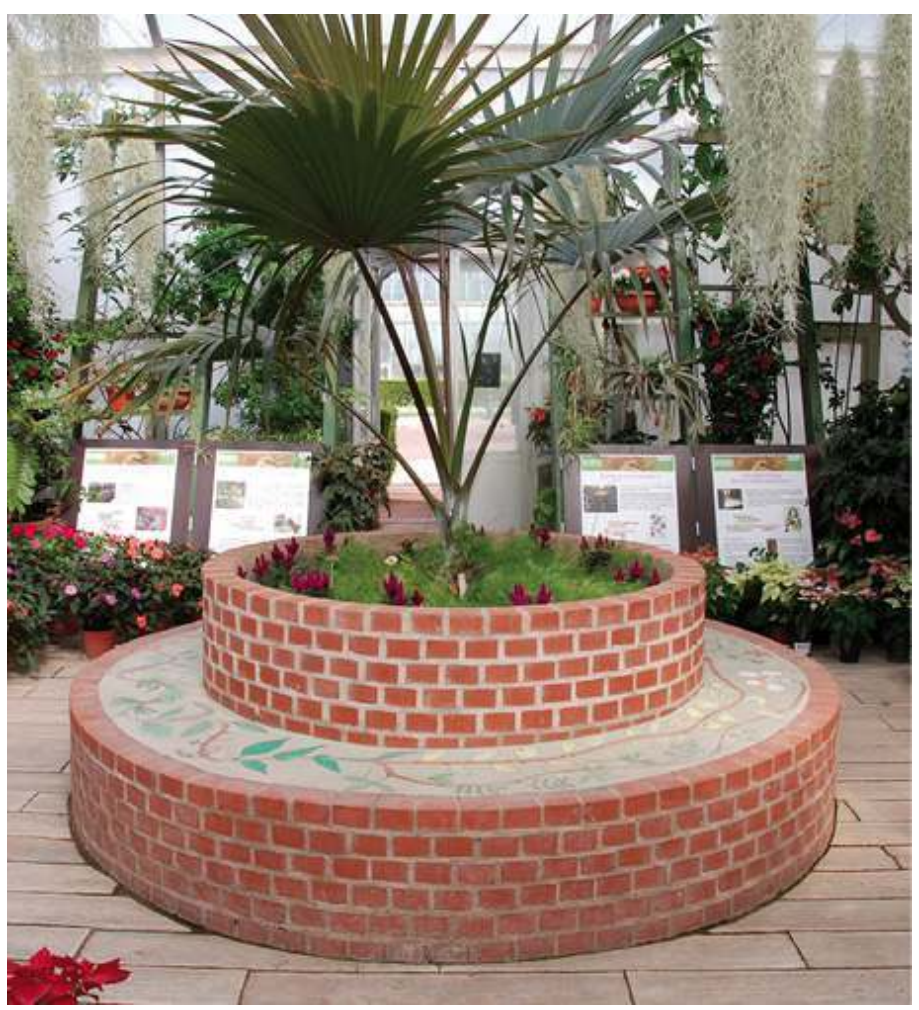

(c) Jardin botanique de Lyon

En fait, l'enquête a surtout montré que le Jardin botanique était perçu plutôt comme une institution scientifique, élitiste. Cette perception venait en partie de nos collections de plantes peu connues du public et de l'emploi systématique (mais obligatoire) de la nomenclature scientifique pour nommer nos plantes. De même, elle a montré que nos visiteurs se contentaient de venir une seule fois, à une saison donnée, alors que la visite, à travers la succession des floraisons, est différente chaque jour. Mais avec cette enquête, nous nous sommes surtout rendu compte que ceux qui connaissent le mieux le jardin et ses potentialités en termes de médiation, de recherche ne sont autres que ceux qui y travaillent. Nous avons donc été assez déçus sur ce point par les conclusions de l'enquête.

\section{Aujourd'hui avez-vous déjà une vision globale claire qui se dessine?}

Le but est vraiment de donner du sens au patrimoine exceptionnel que nous gérons au sein du Parc de la Tête d'Or et de la Ville de Lyon et de voir comment le jardin peut trouver sa place dans le dispositif actuel: un jardin au service des Lyonnais. Une collectivité territoriale comme la Ville de Lyon n'a pas dans sa vocation première de s'impliquer directement dans la recherche fondamentale. Contrairement à l'université, nous n'avons pas vocation à faire de la recherche scientifique. En revanche, notre démarche est de se demander en quoi nos collections vivantes, nos infrastructures et nos savoir-faire peuvent aider la population lyonnaise et comment être un établissement actif et important dans le dispositif municipal. 
Pour faire simple, nous souhaitons présenter le visage d'un jardin botanique au service de la population. À quoi servent les plantes que nous abritons? Sont-elles utiles à la recherche scientifique, à la sauvegarde de la biodiversité, à la dimension touristique du jardin, à la dimension éducative pour les scolaires notamment? Telles sont nos interrogations et le niveau de réflexion auquel nous nous prêtons dans le cadre de nos activités.

\section{Dans cette optique, quelles sont les nouvelles actions que vous souhaitez mettre en place à destination des publics? Quelles sont notamment les thématiques d'exposition envisagées et dans quel espace?}

Depuis 2000, nous avons programmé une vingtaine d'expositions. Certaines étaient entièrement mises en œuvre en interne, aussi bien en termes de conception que de réalisation. Dans d'autres cas, comme l'exposition Hommes, plantes, insectes, regards croisés, nous avons rassemblé une quarantaine de partenaires de l'agglomération lyonnaise et proposé 160 activités en 3 mois (conférences, ateliers, visites, émission de radio...). Chaque thème avait un lien très direct avec le monde végétal (botanique ou horticole), la relation homme-plante ou l'écologie végétale.

Un site Internet pour toute la famille.

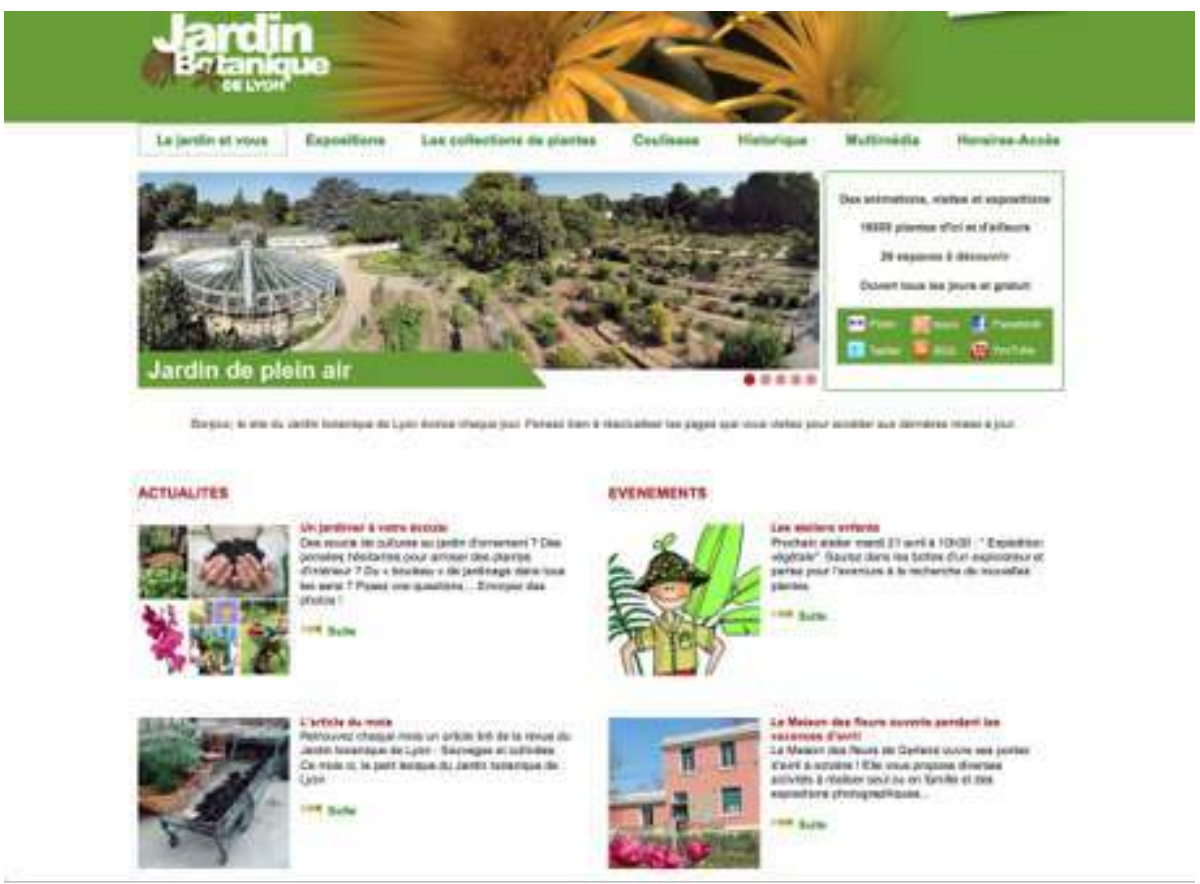

(c) Jardin botanique de Lyon

29 À l'avenir, nous allons nous orienter vers de la médiation culturelle en abordant des thèmes plus larges, tels que les actions éco-citoyennes, le Développement durable, le recyclage des déchets, les grands problèmes de la planète, le changement climatique, le débat sur les OGM... On se rend compte qu'un établissement municipal comme le nôtre, le 
Jardin botanique, à travers ses dizaines de milliers de visiteurs, son accès gratuit et peu « institutionnel », est un lieu idéal pour capter une large partie de la population, aborder ces sujets et assurer une médiation et/ou sensibilisation de la population à ces problématiques. Nous avons constaté cette approche à l'étranger et je souhaite que l'on s'approprie cette idée. Dans le même ordre d'idée, nous souhaitons renforcer encore nos liens avec le tissu local et mettre en œuvre plus de partenariat avec des structures publiques ou privées travaillant autour du végétal. Ce type de partenariat participe au rayonnement du jardin auprès de la population. Ces rapprochements sont souvent très enrichissants pour nos équipes.

La serre hollandaise.

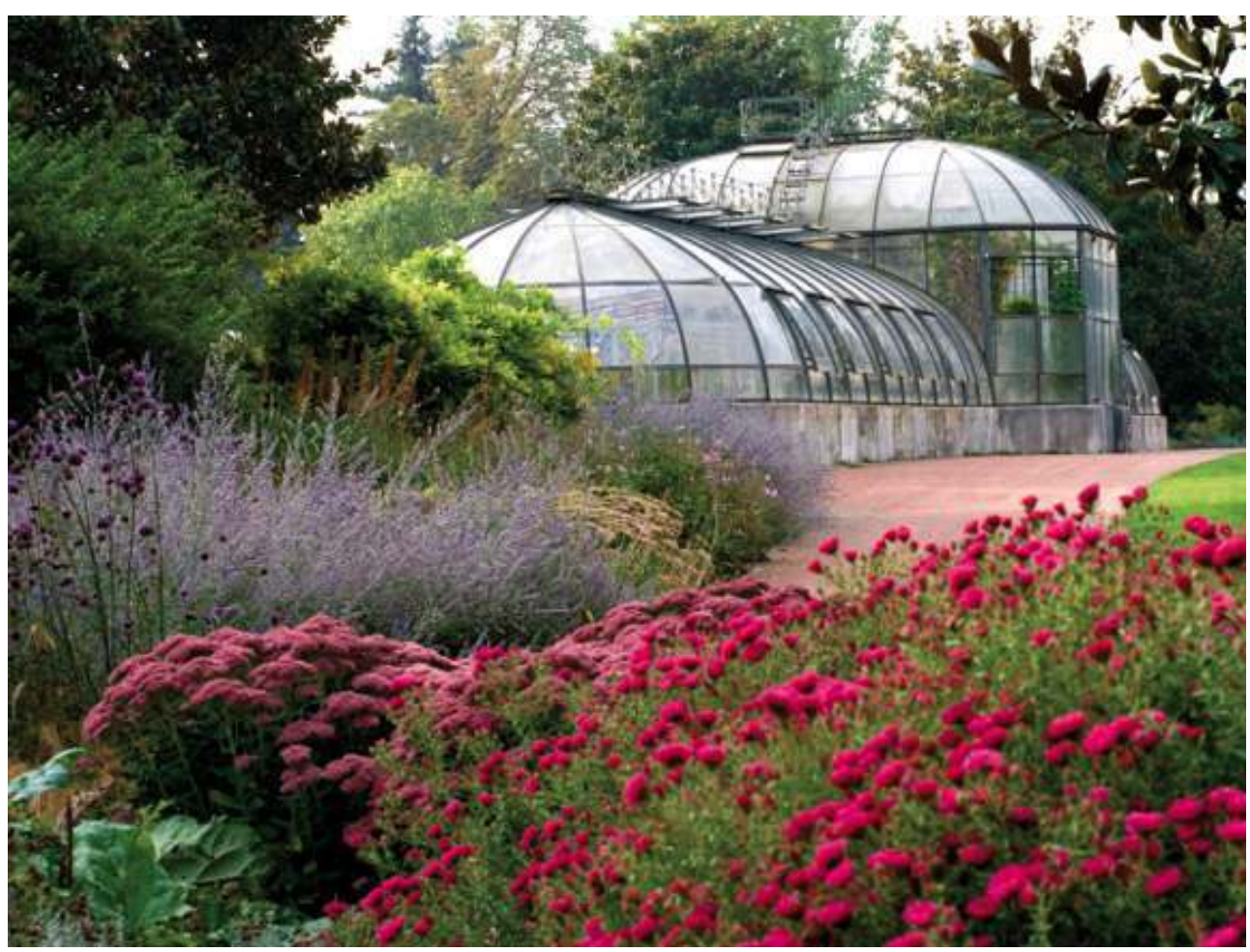

(C) Jardin botanique de Lyon

\section{Cette recherche de rationalisation dans la gestion de vos collections ne menace-t-elle pas votre mission de conservation?}

Non, pas du tout, mais nos collections ont beaucoup évolué depuis notre entrée dans ce plan d'action. D'abord, si nous nous définissons comme un musée, il faut savoir que l'entretien d'un patrimoine vivant représente une activité extrêmement chronophage et un coût très important par rapport à des collections traditionnelles. Dans le coût d'entretien d'une collection botanique de 15500 plantes, $90 \%$ du budget passe dans l'entretien courant de ce vivant (frais de personnel, chauffage, maintien des structures...). Il faut continuer à entretenir ces collections mais pas simplement dans une démarche d'accumulation pure, de " collectionnite ». Il faut à mon sens adosser cette logique à une recherche d'utilité en matière de recherche, d'éducation au Développement durable, de 
conservation... Le but de nos actions consiste donc à essayer de donner du sens à notre travail.

Il y a un vrai débat dans le réseau des jardins botaniques à ce sujet. Ainsi, certains estiment que cela ne sert plus à rien d'avoir des milliers de plantes dans un jardin. Je ne suis pas d'accord avec cette vision. Je pense au contraire qu'il y a encore aujourd'hui une vraie légitimité à conserver des collections importantes. Ne serait-ce que pour confronter les connaissances des ouvrages aux observations faites sur le terrain et continuer à capitaliser l'information sur telle ou telle espèce. La culture de plantes rares ou peu communes par des agents ayant un grand savoir-faire permet, via une organisation bien construite, de capitaliser de nombreuses expériences et connaissances qui seront très utiles dans l'avenir (données sur la biologie, iconographies, techniques de multiplications et conservations).

\section{Quel est le positionnement du jardin botanique par rapport aux autres établissements culturels de la Ville et de la Métropole, notamment le musée des Confluences?}

Nous ne nous situons pas dans le même cadre physique ni la même approche. Je dirais que nous sommes en complémentarité. Nous comptons plusieurs coopérations avec les institutions muséales de la Ville de Lyon comme le musée Gadagne, le musée des BeauxArts, le musée de l'Imprimerie ou encore l'auditorium ou l'Opéra national de Lyon. Par le passé, nous avons présenté des expositions communes avec le muséum de Lyon (actuel musée des Confluences). Il est vrai qu'aujourd'hui, depuis que le musée des Confluences a ouvert, nous n'avons pas à ce jour de projets communs. Pour autant, ce serait tout à fait envisageable et pertinent.

\section{Quelles sont les nouvelles actions à venir pour le Jardin botanique?}

Dans l'immédiat et si le plan d'investissement de la Ville de Lyon est voté, le prochain projet concerne la mise en adéquation des infrastructures du jardin avec ses missions. Le projet prioritaire est la rénovation des petites serres tropicales.

Concernant les rendez-vous, le Jardin botanique participera au $17^{e}$ Congrès mondial des Sociétés de Roses en juin prochain. Cet événement est un bon exemple de ce que sont les missions modernes d'un jardin botanique. À partir de nos collections, nous avons travaillé avec des spécialistes pour définir un plan de collection. À titre d'exemple, nos axes ont été d'identifier les rosiers géniteurs de toutes les roses modernes et de les présenter au public et de mettre en culture les roses lyonnaises les plus emblématiques et les plus rares. C'est ce travail de recherche de pertinence que nous présenterons durant le congrès. Une exposition présentée d'avril à juillet 2015 dans notre orangerie viendra compléter notre offre pour la durée de l'événement. 


\section{RÉSUMÉS}

À l'image des muséums d'Histoire naturelle qui ont amorcé leur transformation dans les années 1980, les jardins botaniques ont entamé leur mutation au début des années 2000. C'est le cas à Lyon, où, depuis plusieurs années, un important chantier de rénovation et réorganisation amène le jardin botanique à se façonner un visage innovant et une identité forte. Frédéric Pautz, son directeur depuis plus de 15 ans, dessine les contours de ce musée vivant du vivant.

INDEX

Mots-clés : jardin botanique

\section{AUTEUR}

\section{ENTRETIEN AVEC FRÉDÉRIC PAUTZ}

directeur du Jardin botanique de Lyon

frederic.pautz@mairie-lyon.fr 